\title{
Do In Seung Gi-Tang extract suppresses adipocyte differentiation in 3T3-L1 cells
}

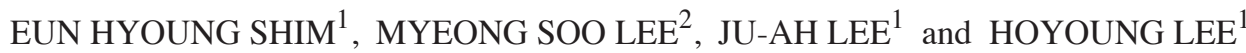 \\ ${ }^{1}$ Korea Medicine Fundamental Research Division and ${ }^{2}$ Clinical Research Division, \\ Korea Institute of Oriental Medicine, Daejeon 305-811, Republic of Korea
}

Received January 21, 2016; Accepted February 10, 2017

DOI: $10.3892 / \mathrm{mmr} .2017 .6446$

\begin{abstract}
Blood stasis syndrome (BSS), additionally called Eohyul, is a basic pathological concept in Traditional Korean Medicine. Do In Seung Gi-Tang (DISGT) is herbal medicine used for the treatment of BSS. It primarily treats metabolic diseases (MDs) including obesity, hypertension, diabetes mellitus and gynecological diseases, by promoting blood circulation. The present study aimed to investigate the anti-adipogenesis effect of DISGT in 3T3-L1 adipocytes using Oil Red O staining, and assessing levels of triglycerides (TGs) and leptin in adipocytes by ELISA and western blot analysis. The results demonstrated that DISGT treatment had inhibitory effects on fat droplet formation, TG accumulation, leptin production and cytokine content, during 3T3-L1 adipocyte differentiation, without affecting cell viability. Additionally, DISGT treatment significantly suppressed the protein expression levels of peroxisome proliferator-activated receptor $\gamma$ and CAAT/enhancer binding protein $\alpha$. These results provide evidence that DISGT has anti-adipogenesis effects on preadipocytes and adipocytes by significantly blocking adipocyte differentiation and lipid accumulation, and suppressing adipogenic gene expression. Therefore, the present study demonstrated the potential of DISGT as a therapeutic agent for the treatment of MDs.
\end{abstract}

\section{Introduction}

Metabolic diseases (MDs), including obesity, elevated triglyceride (TG) levels, reduced levels of high-density lipoprotein cholesterol, elevated blood pressure and impaired glucose metabolism, greatly increase the risk of atherosclerotic cardiovascular disease, hyperlipidemia, hypertension and

Correspondence to: Dr Hoyoung Lee, Korea Medicine Fundamental Research Division, Korea Institute of Oriental Medicine, 483 Expo-Ro, Daejeon 305-811, Republic of Korea E-mail: lhoyoung@kiom.re.kr

Key words: Do In Seung Gi-Tang, blood stasis syndrome, Eohyul, anti-adipogenesis, herbal medicine type 2 diabetes mellitus $(1,2)$. Obesity due to adipocyte hypertrophy leads to alterations in adipocytokine profiles involved in the development of insulin resistance, and in the production of signaling molecules, including peroxisome proliferator-activated receptor (PPAR) $\gamma$, adipocyte protein 2 and leptin. Specifically, PPARs, ligand-activated nuclear transcription factors, regulate gene expression associated with adipocyte differentiation, lipogenesis and glucose metabolism. Furthermore, PPARs are involved in an array of MDs, including type 2 diabetes mellitus, obesity, hyperlipidemia, atherosclerosis and cardiovascular disease (3-5).

Blood stasis syndrome (BSS) is an important pathological concept in Traditional Korean Medicine (TKM). BSS refers to the pathological stagnation of blood circulation, delayed blood flow, dysfunction of endothelial cells or metabolic disorder. It was first recorded in Huangdi's Inner Classic (6). Numerous studies have reported that BSS is associated with MDs, including atherosclerosis, hypertension, coronary artery lesions, cardiac function, blood lipid, diabetes mellitus and insulin resistance (7-9).

The properties of Do In Seung Gi-Tang (DISGT), a traditional herbal formula used to treat BSS, have been recorded in the Dongui Bogam (10). It is primarily used in TKM for treating obesity and hypertension, diabetes mellitus, inflammation, immunity and gynecological diseases, including menstrual irregularity, by promoting blood circulation (11-13).

Although clinical studies have assessed the symptom relief effects of DISGT treatment, few studies have investigated the underlying mechanism of DISGT in the pathology or biology of adipocytes. As a result, this underlying mechanism remains unclear. Therefore, the present study investigated the potential anti-adipogenesis effect of DISGT on 3T3-L1 adipocyte differentiation and regulation of protein expression associated with lipid metabolism.

\section{Materials and methods}

Materials. The 3T3-L1 mouse fibroblast cell line was purchased from the American Type Culture Collection (Manassas, VA, USA). Dulbecco's modified Eagle's medium (DMEM), fetal bovine serum (FBS), penicillin-streptomycin (P\&S), bovine calf serum (NCS) and Dulbecco's phosphate-buffered saline (DPBS) were obtained from Gibco; Thermo Fisher Scientific, Inc. (Waltham, MA, USA). 
Dimethyl sulfoxide (DMSO), formaldehyde, dexamethasone (DEX), 3-isobutyl-1-methylisobutylxanthine (IBMX), insulin, triton X-100 and Oil Red O staining powder were obtained from Sigma-Aldrich; Merck Millipore (Darmstadt, Germany). Cell Counting kit-8 (CCK-8) was purchased from Dojindo Molecular Technologies, Inc. (Kumamoto, Japan). The EnzyChrom ${ }^{\mathrm{TM}}$ TG assay kit was purchased from BioAssay Systems (Hayward, CA, USA) and the Mouse/Rat Leptin Quantakine ELISA kit (cat. no. MOB00) was purchased from R\&D Systems, Inc. (Minneapolis, MI, USA). A MILLIPLEX ${ }^{\circledast}$ MAP Mouse Adipocyte Magnetic Bead Panel kit was obtained from EMD Millipore (Billerica, MA, USA). The P38 inhibitor SB203580 (cat. no. 5633) was purchased from Cell Signaling Technology, Inc. (Danvers, MA, USA). Mouse anti-PPAR $\gamma$ (cat. no. sc-7273), rabbit anti-CCAAT/enhancer binding protein $(\mathrm{C} / \mathrm{EBP}) \alpha$ (cat. no. sc-61) and mouse anti- $\beta$-actin (cat. no. sc-81178) primary antibodies were purchased from Santa Cruz Biotechnology, Inc. (Dallas, TX, USA). Horseradish peroxidase (HRP)-conjugated secondary antibodies, goat anti-rabbit IgG (cat. no. 170-6515) and goat anti-mouse IgG (cat. no. 170-6516), were obtained from Bio-Rad Laboratories, Inc. (Hercules, CA, USA). All other reagents from commercial sources were of analytical grade.

Preparation of herbal extracts. DISGT was prepared by extracting the five different types of herb listed in Table I. Each herb, including DISGT, was purchased from Omniherb (Daegu, Korea) in 2012. A voucher specimen (BS-1) was stored at the Korea Medicine Fundamental Research Division, Korea Institute of Oriental Medicine (Daejeon, Korea). The extract was crushed using a grinder and extracted using distilled water. The solution was filtered through cotton and extracted at $100^{\circ} \mathrm{C}$ for $3 \mathrm{~h}$ using a COSMOS-660 Vacuum Extractor (Kyungseo E\&P, Incheon, Korea), following which it was concentrated using a vacuum evaporator (EYELA N-12; EYELA CA-1112; Tokyo Rikakikai Co., Ltd., Tokyo, Japan). The extract was freeze-dried (PVTFD-100; ilShin BioBase Co., Ltd., Dongducheon, Korea) to create a powder (extraction yield, $26.63 \%$ ). The prepared powder was stored at $-70^{\circ} \mathrm{C}$ until further use.

Cell culture and differentiation. For cell culture and differentiation, 3T3-L1 preadipocytes were grown and seeded into 6-well plates containing DMEM supplemented with $10 \% \mathrm{NCS}$ and $1 \% \mathrm{P} \& \mathrm{~S}$ at $37^{\circ} \mathrm{C}$ in a humidified atmosphere of $5 \% \mathrm{CO}_{2}$. Once cells had reached confluency (day 0) they were exposed to differentiation medium: DMEM supplemented with $10 \% \mathrm{FBS}, 1 \% \mathrm{P} \& \mathrm{~S}$, and a mixture of $0.5 \mathrm{mM}$ IBMX, $1 \mu \mathrm{M}$ DEX and $1 \mu \mathrm{g} / \mathrm{ml}$ insulin (MDI), and were treated with $62.5,125,250$ or $500 \mu \mathrm{g} / \mathrm{ml}$ DISGT, and $10 \mu \mathrm{M}$ of $\mathrm{SB} 203580$, which is a 38 mitogen-activated protein kinase (MAPK) inhibitor and was used as a positive control to confirm the efficacy of DISGT, for $48 \mathrm{~h}$ (from days 0 to 2). Cells were subsequently maintained in post-differentiation medium: DMEM containing $1 \mu \mathrm{g} / \mathrm{ml}$ insulin in the absence of IBMX or DEX, and subsequently treated with various concentrations of DISGT and $10 \mu \mathrm{M} \mathrm{SB} 203580$ for $72 \mathrm{~h}$ (from days 2 to 5). Following this, the medium was replaced with post-differentiation medium DMEM and cells were treated with various concentrations of DISGT and $10 \mu \mathrm{M}$ SB203580 for $48 \mathrm{~h}$ (from days 5 to 7 ).

Cell cytotoxicity. The effect of DISGT on cell viability was determined by a CCK-8 assay. 3T3-L1 cells were seeded at a density of $1.5 \times 10^{3}$ cells into 48 -well plates. After 2 days, cells reached confluency and were treated with $0,10,20,50,100$, 200,500 or $1000 \mu \mathrm{g} / \mathrm{ml}$ DISGT for 7 days. Following incubation with DISGT for 7 days, cells were treated with CCK-8 and incubated at $37^{\circ} \mathrm{C}$ for $4 \mathrm{~h}$. Following treatment, absorbance was measured at a wavelength of $450 \mathrm{~nm}$ using a Benchmark Plus microplate reader (Bio-Rad Laboratories, Inc.). Data were calculated as percentage of cell viability compared with control cells.

Oil Red O staining. To observe the fat droplets in adipocytes, cells were stained with Oil Red O on day 7 following differentiation induction. Cells treated with various concentrations of DISGT were washed twice with DPBS and fixed with $10 \%$ formalin for $30 \mathrm{~min}$. Following this, cells were stained with $0.3 \%$ Oil Red $\mathrm{O}$ in $60 \%$ isopropanol for $1 \mathrm{~h}$ at room temperature. Cells were subsequently washed three times with distilled water and imaged using a light microscope (Olympus Corporation, Tokyo, Japan). Fat droplets stained with Oil Red O solution were dissolved with $100 \%$ DMSO and quantified by measuring the optical absorbance at a wavelength of $530 \mathrm{~nm}$ using a Benchmark Plus microplate reader.

$T G$ and leptin production. TG and leptin contents were measured in differentiated 3T3-L1 cells on day 7. A TG analysis was performed on lipid droplets collected from each sample. TGs were quantified using an EnzyChrom ${ }^{\mathrm{TM}}$ TG assay according to the manufacturer's protocol and a colorimetric detection method (absorbance measured at a wavelength of $570 \mathrm{~nm}$ ) (14). Leptin was quantified using a mouse leptin-specific ELISA kit according to the manufacturer's protocol. The detection limit of the assay was typically $<22 \mathrm{pg} / \mathrm{ml}(15)$.

Adipocyte detection. A MILLIPLEX MAP Mouse Adipocyte Magnetic Bead Panel kit was used to detect adipocytes in the supernatant. Measurements of adipocyte concentrations were conducted according to the manufacturer's protocol. A total of seven standard samples were set; two quality controls and the standard samples were run in duplicate. Detection samples were assayed in triplicate. Signal values were detected on a Bio-Plex ${ }^{\circledR} 200$ system and Bio-Plex Pro II Wash Station (xMAP ${ }^{\circledR}$ Technology; Luminex Corporation, Austin, TX, USA). Briefly, the binding of specific factors begins in the bead mixture suspended with a detection sample. Following an overnight incubation at $4^{\circ} \mathrm{C}$, a biotinylated detection antibody was introduced. A subsequent incubation with a streptavidin-phycoerythrin conjugate was performed to complete the reaction on the microplates. Finally, each assay was analyzed using a Bio-Plex 200 system. Concentrations were calculated according to a standard curve.

Protein preparation and western blot analysis. Cells were washed and harvested with ice-cold DPBS. Lysates were prepared using radioimmunoprecipitation cell lysis 
Table I. Composition of Do In Seung Gi-Tang.

\begin{tabular}{|c|c|c|}
\hline \multicolumn{2}{|c|}{ Compound } & \multirow[b]{2}{*}{ Dosage $(\mathrm{g})$} \\
\hline Herbal name & Scientific name & \\
\hline Rhai Rhizoma & $\begin{array}{l}\text { Rheum officinale } \\
\text { Baillon }\end{array}$ & 12.00 \\
\hline Persicae semen & $\begin{array}{l}\text { Prunus persica } \\
\text { Batsch }\end{array}$ & 2.00 \\
\hline Cinnammomi & Cinnamomum & 8.00 \\
\hline Cortex & loureirii Nees & \\
\hline Natrii Sulfas & Conyza canadensis & 8.00 \\
\hline Glycyrrhizae & Glycyrrhiza uralensis & 4.00 \\
\hline Radix & Fisch & \\
\hline
\end{tabular}

Total, $34 \mathrm{~g}$; yield, $26.63 \%$.

buffer containing $0.5 \mathrm{M}$ Tris- $\mathrm{HCl}(\mathrm{pH} 7.4), 1.5 \mathrm{M} \mathrm{NaCl}$, $2.5 \%$ deoxylcholic acid, $10 \%$ NP-40 and $10 \mathrm{mM}$ EDTA. Protein concentration was measured using a Bicinchoninic Acid Protein assay kit (Thermo Fisher Scientific Inc.). Cell lysates $(30 \mu \mathrm{g})$ were separated by $4-20 \%$ Criterion $^{\mathrm{TM}}$ TGX $^{\mathrm{TM}}$ precast gel (Bio-Rad Laboratories, Inc.) electrophoresis and subsequently transferred onto polyvinylidene difluoride membranes (GE Healthcare Life Sciences, Chalfont, UK). Membranes were blocked with 5\% skimmed milk and incubated overnight at $4^{\circ} \mathrm{C}$ with $\mathrm{C} / \mathrm{EBP} \alpha, \operatorname{PPAR} \gamma$ and $\beta$-actin primary antibodies at a dilution of 1:1,000. After $1 \mathrm{~h}$ incubation at room temperature with HRP-conjugated anti-mouse for or anti-rabbit secondary antibodies $(1: 3,000)$, protein bands were detected with an Enhanced Chemiluminescence assay kit (Thermo Fisher Scientific, Inc.). Images were captured using a ChemiDoc ${ }^{\mathrm{TM}}$ XRS+ image analyzer (Bio-Rad Laboratories, Inc.). Relative density of $\mathrm{C} / \mathrm{EBP} \alpha$ and PPAR $\gamma$ were normalized to $\beta$-actin and quantified using Image $\mathrm{Lab}^{\mathrm{TM}}$ software version 4.0 (Bio-Rad Laboratories, Inc.).

Statistical analysis. Data are presented as the mean + standard error $(n=3)$ and were compared using one-way analysis of variance followed by a Bonferroni correction, in SPSS software version 13.0 (SPSS, Inc., Chicago, IL, USA). $\mathrm{P}<0.05$ was considered to indicate a statistically significant difference.

\section{Results}

Herbal formula and cell viability. The yield of the DISGT water extract was $26.63 \%$ (w/w) following freeze-drying. CCK-8 analysis was performed to evaluate the cytotoxicity of DISGT. DISGT did not demonstrate significant cytotoxicity at $500 \mu \mathrm{g} / \mathrm{ml}$ (data not shown). The present study used DISGT at concentrations of $62.5,125,250$ and $500 \mu \mathrm{g} / \mathrm{ml}$.

Effects of DISGT on intracellular function in differentiated 3T3-L1 adipocytes. To assess the effect of DISGT on adipogenesis, intracellular lipid accumulation was analyzed in mature adipocytes. Following treatment with various concentrations
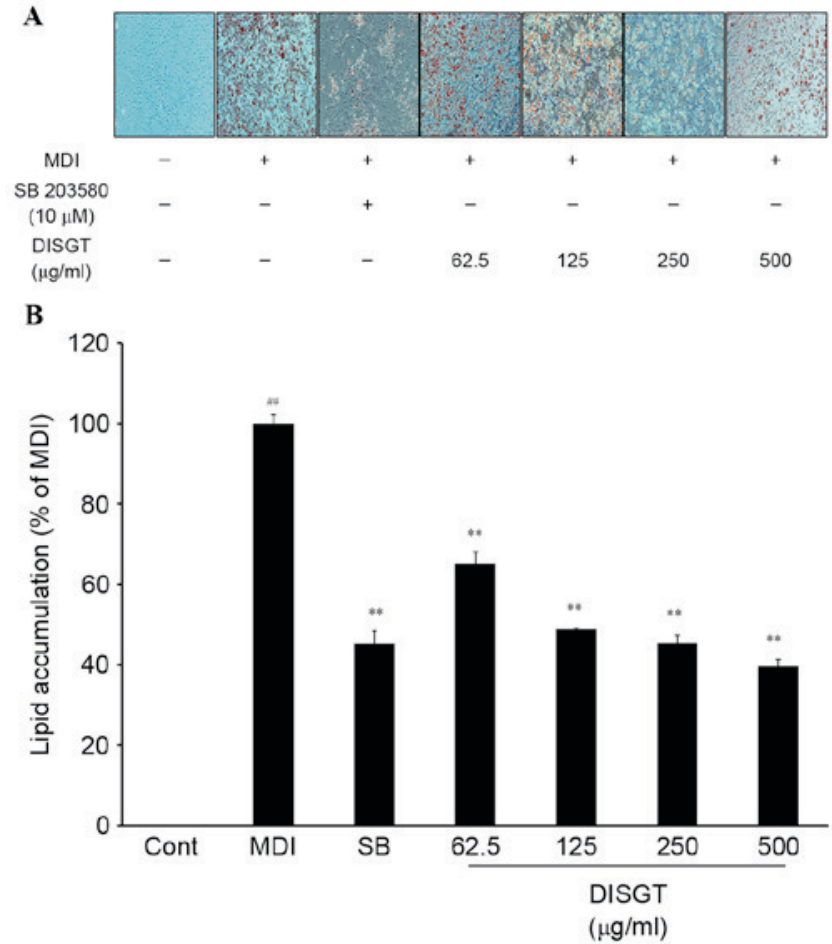

Figure 1. Inhibitory effect of DISGT on lipid accumulation in 3T3-L1 adipocytes. (A) Fat droplets were measured by Oil Red O staining and observed under a microscope (magnification, x100). (B) Lipid droplets were dissolved in dimethyl sulfoxide and quantified by reading the absorbance at a wavelength of $530 \mathrm{~nm}$. Data are presented as the mean + standard error $(\mathrm{n}=3)$. ${ }^{\# \#} \mathrm{P}<0.01$ vs. Cont; ${ }^{* *} \mathrm{P}<0.01$ vs. MDI. DISGT, Do In Seung Gi-Tang; Cont, control; MDI, mixture of 3-isobutyl-1-methylisobutylxanthine, dexamethasone and insulin; SB, SB203580.

of DISGT for 7 days, lipid accumulation was significantly suppressed in a dose-dependent manner (Fig. 1).

For intracellular lipid regulation, TG levels in the lysate and leptin levels in the supernatant were measured. The TG concentration of the control group was $0.0 \pm 4.26 \mu \mathrm{M}$. Following treatment with MDI, the TG levels increased to $158.67 \pm 14.13 \mu \mathrm{M}$. DISGT treatment significantly suppressed TG release in a dose-dependent manner $(\mathrm{P}<0.001)$. SB203580 is a well-established p38 MAPK inhibitor. A previous study demonstrated that when p38 MAPK was inhibited, adipocyte differentiation was inhibited by downregulation of adipogenic-specific transcription factors, including $\mathrm{C} / \mathrm{EBPs}$ and PPAR $\gamma(16)$. SB203580 $(10 \mu \mathrm{M})$, which was used as a positive control, reduced the TG concentration to $36.90 \pm 12.31 \mu \mathrm{M}$ $(\mathrm{P}<0.01$ vs. MDI). DISGT $(500 \mu \mathrm{g} / \mathrm{ml})$ treatment was more effective compared with SB203580, and reduced the TG concentration to $19.32 \pm 6.72 \mu \mathrm{M}(\mathrm{P}<0.01$ vs. MDI; Fig. 2A). To determine the effect of leptin inhibition on adipogenesis in adipocytes, 3T3-L1 adipocytes were treated with MDI. As presented in Fig. 2B, MDI treatment of 3T3-L1 adipocytes significantly increased leptin levels compared with the control $(\mathrm{P}<0.001)$. When MDI-differentiated 3T3-L1 cells were treated with DISGT, leptin release was significantly inhibited in a dose-dependent manner $(\mathrm{P}<0.001)$.

Effects of DISGT on the secretion of adipokines in the 3T3-L1 adipocytes. To examine the effect of DISGT on 
A

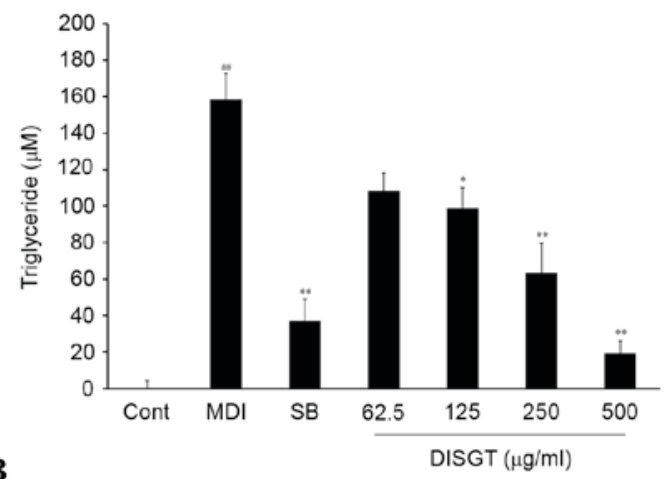

B

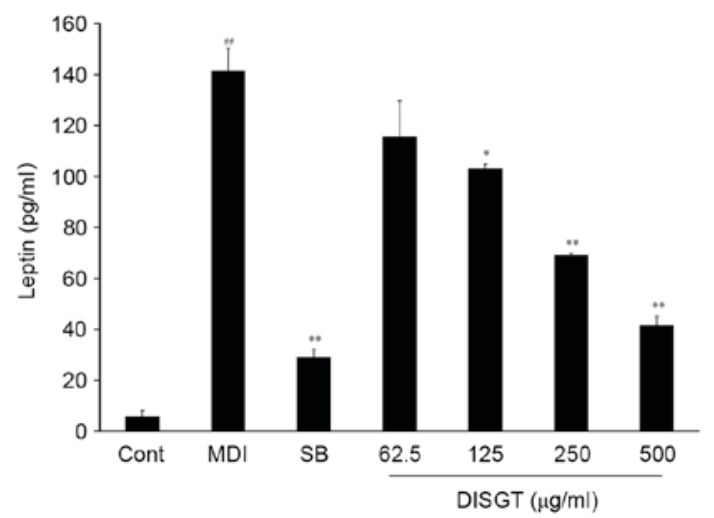

Figure 2. Inhibitory effect of DISGT on TG and leptin production by 3T3-L1 adipocytes. 3T3-L1 preadipocytes were differentiated into adipocytes and treated with $0,62.5,125,250$ or $500 \mu \mathrm{g} / \mathrm{ml}$ DISGT for 7 days. (A) TG production following DISGT treatment. (B) Leptin production following DISGT treatment. Data are presented as the mean + standard error $(\mathrm{n}=3) .{ }^{\# \#} \mathrm{P}<0.01$ vs. Cont; ${ }^{\mathrm{P}}<0.05$ and ${ }^{* *} \mathrm{P}<0.01$ vs. MDI. DISGT, Do In Seung Gi-Tang; TG, triglyceride; Cont, control; MDI, mixture of 3-isobutyl-1-methylisobutylxanthine, dexamethasone and insulin; SB, SB203580.

MDI-differentiated 3T3-L1 adipocytes, a multiplex assay was performed to detect adiponectin (Fig. 3A), resistin (Fig. 3B) and plasminogen activator inhibitor-1 (PAI-1; Fig. 3C) in the cell supernatants. All factors were increased following MDI treatment. DISGT significantly and dose-dependently suppressed the release of adiponectin and resistin $(\mathrm{P}<0.001)$. The positive control SB203580 additionally inhibited release of adiponectin and PAI-1. DISGT treatment suppressed the release of adiponectin and resistin to a greater extent compared with SB203580 $(10 \mu \mathrm{M})$. Furthermore, DISGT treatment inhibited the release of PAI-1 by MDI-differentiated 3T3-L1 adipocytes. DISGT and SB203580 inhibited PAI-1 levels to a similar extent.

Effect of DISGT on the expression of adipocyte-associated proteins during adipogenesis. To determine if DISGT affects adipogenesis during adipocyte differentiation from 3T3-L1 preadipocytes, protein expression of $\mathrm{C} / \mathrm{EBP} \alpha$ and PPAR $\gamma$ were examined by western blotting following treatment with various concentrations of DISGT for 7 days. At 7 days post-incubation, these proteins were dramatically upregulated and expressed in the differentiated 3T3-L1 cells. However, DISGT treatment decreased the expression of C/EBP $\alpha$ and PPAR $\gamma$ compared with cells cultured with MDI alone. The expression of $\mathrm{C} / \mathrm{EBP} \alpha$ and PPAR $\gamma$ were markedly decreased following treatment with $500 \mu \mathrm{g} / \mathrm{ml}$ DISGT (Fig. 4).
A
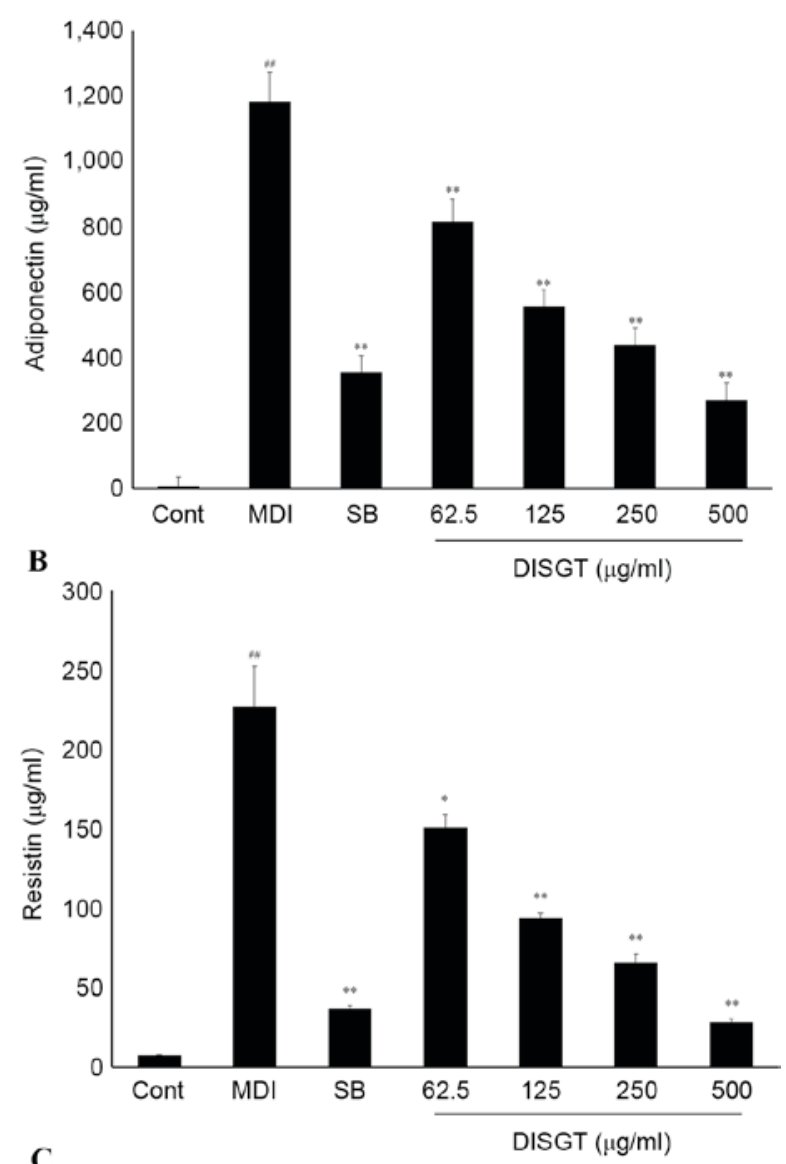

C

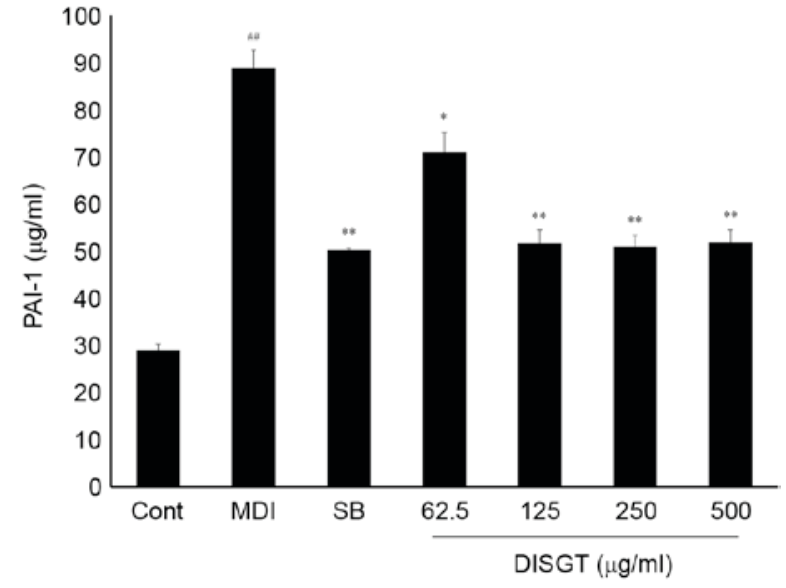

Figure 3. Inhibitory effect of DISGT on adipokine production in 3T3-L1 adipocytes. 3T3-L1 preadipocytes were differentiated into adipocytes and treated with $0,62.5,125,250$ or $500 \mu \mathrm{g} / \mathrm{ml}$ DISGT for 7 days. (A) Adiponectin, (B) resistin and (C) PAI-1 levels were assessed following DISGT treatment. Data are presented as the mean + standard error $(n=3)$. ${ }^{\# \#} \mathrm{P}<0.01$ vs. Cont; ${ }^{*} \mathrm{P}<0.05$ and ${ }^{* *} \mathrm{P}<0.01$ vs. MDI. DISGT, Do In Seung Gi-Tang; PAI-1, plasminogen activator inhibitor-1; Cont, control; MDI, mixture of 3-isobutyl-1-methylisobutylxanthine, dexamethasone and insulin; SB, SB203580.

\section{Discussion}

BSS, known as Eohyul in TKM, refers to stagnation of blood circulation, retained blood and vascular obstruction; studies on its underlying mechanisms and treatment have been performed in Korea and China $(11,12)$. BSS is one of the most common 


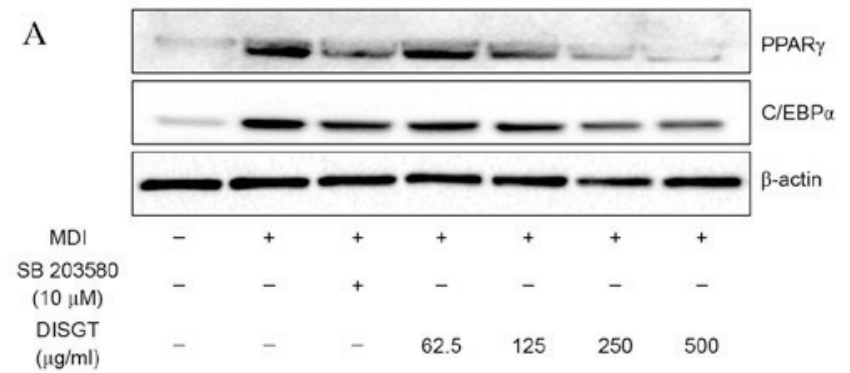

B
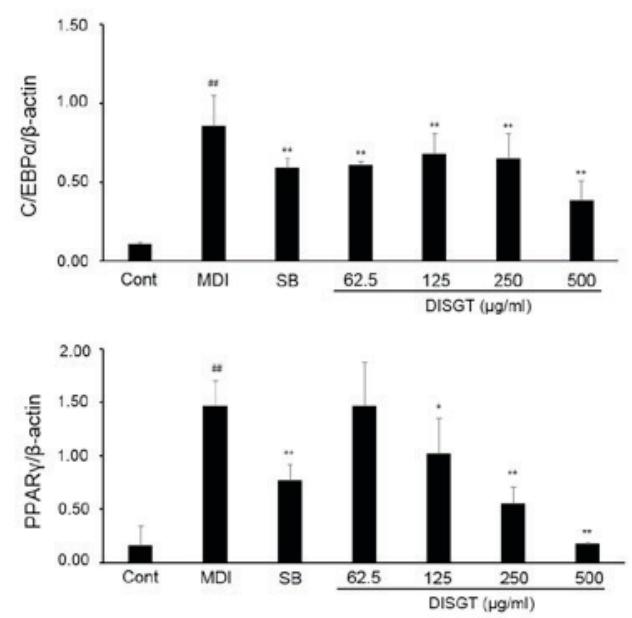

Figure 4. Protein expression levels following DISGT treatment in 3T3-L1 adipocytes at day 7. (A) Representative western blot images of PPAR $\gamma$ and $\mathrm{C} / \mathrm{EBP} \alpha$ protein expression levels in 3T3-L1 adipocytes following treatment with various concentrations of DISGT. $\beta$-actin served as an internal control. DISGT treatment suppressed protein expression levels of PPAR $\gamma$ and $\mathrm{C} / \mathrm{EBP} \alpha$ in 3T3-L1 adipocytes. (B) The graph is the relative density of PPAR $\gamma$ and $\mathrm{C} / \mathrm{EBP} \alpha$ after normalization to $\beta$-actin. Data are presented as the mean + standard error $(n=3) .{ }^{\# \#} \mathrm{P}<0.01$ vs. Cont; ${ }^{*} \mathrm{P}<0.05$ and ${ }^{* *} \mathrm{P}<0.01$ vs. MDI. DISGT, Do In Seung Gi-Tang; PPAR $\gamma$, peroxisome proliferator-activated receptor $\gamma ; \mathrm{C} / \mathrm{EBP} \alpha, \mathrm{CAAT} / \mathrm{enhancer}$ binding protein $\alpha$; Cont, control; MDI, mixture of 3-isobutyl-1-methylisobutylxanthine, dexamethasone and insulin; SB, SB203580.

illnesses in TKM and is associated with MDs, including obesity, atherosclerotic cardiovascular disease, hyperlipidemia, hypertension and type 2 diabetes mellitus (17). Obesity is characterized by an excess of body fat and adipose tissue and is caused by an imbalance between calorie intake and usage. It involves MDs and chronic inflammatory responses (18-21).

The present study detected an anti-adipogenesis effect of DISGT treatment on MDI-differentiated 3T3-L1 adipocytes. DISGT, an agent that has been used to treat various symptoms associated with BSS in TKM, consists of five types of herbal ingredients. It is considered to improve blood circulation by removing blood stasis. Furthermore, it has been investigated for the treatment of various clinical conditions, including atherosclerotic disease, gynecological disease, diabetes mellitus and chronic pyelonephritis, by promoting blood flow (22-24). Therefore, the present study focused on the anti-adipogenesis mechanism associated with adipokine levels and intracellular-associated factors, including TG, leptin, adiponectin, resistin and PAI-1. The results revealed that $0-1,000 \mu \mathrm{g} / \mathrm{ml}$ DISGT was not cytotoxic to adipocytes. Oil Red $\mathrm{O}$ staining was used to visualize the TG-containing fat droplets in adipocytes; DISGT treatment significantly reduced the fat droplets in MDI-differentiated 3T3-L1 adipocytes. DISGT treatment significantly inhibited the release of certain factors associated with adipokines, including leptin, $\mathrm{TG}$, adiponectin and resistin in a dose-dependent manner, however, this was not observed for PAI-1, and inhibited the PPAR $\gamma$ signaling pathway. These results demonstrated that DISGT treatment dose-dependently inhibited differentiation and lipid accumulation in 3T3-L1 adipocytes.

Pathological adipocyte differentiation markedly increases the level of adipocytokines, or adipokines, including tumor necrosis factor- $\alpha$, leptin, adiponectin and resistin, which affects systemic energy balance (25). Therefore, inhibiting adipocyte differentiation may be important as a therapeutic approach for the treatment and prevention of MDs related to obesity.

Together with high blood pressure and reduced high-density lipoprotein levels, elevated serum TG levels are a primary marker of obesity (26). Lipid formation in adipose tissue occurs at a late stage during adipogenesis and is associated with elevated TG levels and adipose tissue mass $(27,28)$. In the present study, DISGT treatment markedly suppressed morphological differentiation and TG accumulation by inhibiting fat droplet formation during 3T3-L1 adipocyte differentiation.

Leptin concentrations in humans and rodents have been closely associated with adiposity and body weight alterations. Furthermore, plasma leptin has been demonstrated to be highly correlated with body mass index in rodents and obese humans (29). As a hormone, it links lipid storage, regulates body weight and is influenced by environmental factors or other hormones, inluding MDI and DEX. Blood leptin levels are known to be closely connected with TG levels and the quantity of adipose tissue in the body $(30,31)$. The present study demonstrated that DISGT treatment significantly suppressed TG and leptin concentrations. These results suggested that DISGT may have a biological role in decreasing lipid formation and TGs and acting as a negative regulator of adipogenesis.

In the present study, DISGT treatment inhibited adiponectin, resistin and PAI-1 levels in differentiated 3T3-L1 cells. Reduced circulating adiponectin concentrations are associated with insulin resistance and MDs (32). Resistin, additionally named adipocyte secreted factor, is a member of the resistin-like molecules family of proteins with cysteine-rich structures. Resistin concentrations are increased in numerous inflammation-associated disorders, including atherosclerosis, chronic inflammatory bowel disease, chronic renal disease and arthritis (33).

$\mathrm{C} / \mathrm{EBP} \beta$ and $\mathrm{C} / \mathrm{EBP} \delta$ are rapidly triggered by hormones including DEX and methylisobutylxanthine during early adipocyte differentiation. Expression levels of these proteins are subsequently reduced, and expression of $\mathrm{C} / \mathrm{EBP} \alpha$ and PPAR $\gamma$ promptly increase in the mid and late stages of adipogenesis (34-36). PPAR $\gamma$ is a critical transcription factor markedly expressed in adipose tissue that stimulates adipocyte differentiation.Furthermore, it is a key nuclear receptor thatregulates an array of diverse functions in numerous cell types, including the regulation of genes associated with growth and differentiation (37). Its primary function is to directly regulate the development of adipose tissue, which involves coordinating the expression of a variety of genes responsible for the formation of the mature 
adipocyte phenotype (5). C/EBP $\alpha$ and PPAR $\gamma$ individually or in combination regulate the activation of adipose-specific genes critical to the development of the adipose phenotype and adipose biosynthesis, including fatty acid synthase, acetyl-coenzyme A synthetase 1 and fatty acid binding protein (38).

The present study investigated alterations in the protein expression levels of C/EBP $\alpha$ and PPAR $\gamma$ following adipocyte differentiation for 7 days to evaluate the effects of DISGT treatment on the activity of these adipogenesis-associated transcription factors. DISGT treatment significantly suppressed MDI-induced protein expression levels of C/EBP $\alpha$ and PPAR $\gamma$, which are primary markers of adipogenesis. These results demonstrated that DISGT treatment significantly blocked adipocyte differentiation and lipid accumulation by suppressing adipogenic gene expression. Thus, the present study suggested the potential of DISGT as a therapeutic agent for the treatment of MDs associated with BSS.

\section{Acknowledgements}

The present study was supported by the Korea Institute of Oriental Medicine (grant no. K16110).

\section{References}

1. Eckel RH, Grundy SM and Zimmet PZ: The metabolic syndrome. Lancet 365: 1415-1428, 2005.

2. Kaur J: A comprehensive review on metabolic syndrome. Cardiol Res Pract 2014: 943162, 2014.

3. Shin SS, Jung YS, Yoon KH, Choi S, Hong Y, Park D, Lee H, Seo BI, Lee HY and Yoon M: The Korean traditional medicine gyeongshingangjeehwan inhibits adipocyte hypertrophy and visceral adipose tissue accumulation by activating PPARalpha actions in rat white adipose tissues. J Ethnopharmacol 127 47-54, 2010.

4. Pakala R, Kuchulakanti P, Rha SW, Cheneau E, Baffour R and Waksman R: Peroxisome proliferator-activated receptor gamma: Its role in metabolic syndrome. Cardiovasc Radiat Med 5: 97-103, 2004.

5. Farmer SR: Regulation of PPARgamma activity during adipogenesis. Int J Obes (Lond) 29 (Suppl 1): S13-S16, 2005.

6. Li SM, Xu H and Chen KJ: The diagnostic criteria of blood-stasis syndrome: Considerations for standardization of pattern identification. Chin J Integr Med 20: 483-489, 2014.

7. Zhou XQ, Liang H, Sun X and Zhou HT: Correlation between TCM blood stasis pattern of coronary heart disease and coronary angiography result: A meta-analysis. Chin J Evid Based Med 12: 1470-1477, 2012.

8. Ji W, Zhou J, Wang S and Ji Z: Logistic regression analysis of relevance between different blood stasis syndromes and related factors in angina pectoris patients. Chin Arch Tradit Chin Med 7: 040, 2013 (In Chinese).

9. Yu X, Zhang $\mathrm{L}$ and $\mathrm{Xu} \mathrm{H}$ : Progress in research on relevant factors affecting TCM syndrome differentiation of CHD. Chin J Int Med Cardio/Cerebrovasc Dis 7: 581-584, 2009 (In Chinese).

10. Heo J: Donguibogam. Namsandang Publ. Corp., Seoul, p462, 2014.

11. Lee YJ, Kim EK, Kim HY, Yoon JJ, Lee SM, Lee GM, Kang DG and Lee HS: Therapeutic effect of doinseunggi-tang on diabetic vascular dysfunction. HFS 21: 119-130, 2013.

12. Kosuge T, Ishida $H$ and Ishii M: Studies on active substances in the herbs used for oketsu ('stagnant blood') in Chinese medicine. II. On the anticoagulative principle in persicae semen. Chem Pharm Bull (Tokyo) 33: 1496-1498, 1985.

13. Ge RY, Zhou CH and She YC: Influences of stigma croci and semen persicae on function of ovary-uterus in pseudopregnant rats. J Tradit Chin Med 3: 23-26, 1983.

14. Orban T, Palczewska G and Palczewski K: Retinyl ester storage particles (retinosomes) from the retinal pigmented epithelium resemble lipid droplets in other tissues. J Biol Chem 286: 17248-17258, 2011.
15. Columba-Cabezas S, Iaffaldano G, Chiarotti F, Alleva E and Cirulli F: Early handling increases susceptibility to experimental autoimmune encephalomyelitis (EAE) in C57BL/6 male mice. J Neuroimmunol 212: 10-16, 2009.

16. Engelman JA, Lisanti MP and Scherer PE: Specific inhibitors of p38 mitogen-activated protein kinase block 3T3-L1 adipogenesis. J Biol Chem 273: 32111-32120, 1998.

17. Chen KJ: Blood stasis syndrome and its treatment with activating blood circulation to remove blood stasis therapy. Chin J Integr Med 12: 891-896, 2012.

18. Spiegelman BM and Flier JS: Adipogenesis and obesity: Rounding out the big picture. Cell 87: 377-389, 1996.

19. Sartipy P and Loskutoff DJ: Monocyte chemoattractant protein 1 in obesity and insulin resistance. Proc Natl Acad Sci USA 100: 7265-7270, 2003.

20. Wofford MR, Andrew ME, Brown A, King D, Pickett RA, Stevens J, Wyatt S and Jones DW: Obesity hypertension in the atherosclerosis risk in communities cohort: Implications of obesity guidelines. J Clin Hypertens 1: 27-32, 1999.

21. Pi-Sunyer FX: The obesity epidemic: Pathophysiology and consequences of obesity. Obes Res 10 (Suppl 2): S97-S104, 2002.

22. Yoon JJ, Lee YJ, Park OJ, Lee SM, Lee YP, Cho NG, Kang DG and Lee HS: Doinseunggitang ameliorates endothelial dysfunction in diabetic atherosclerosis. Evid Based Complement Alternat Med 2013: 783576, 2013.

23. Sung YY, Kim DS, Choi G, Kim SH and Kim HK: Dohaekseunggi-tang extract inhibits obesity, hyperlipidemia, and hypertension in high-fat diet-induced obese mice. BMC Complement Altern Med 14: 372, 2014.

24. Chen Q: Pharmacology and application of Chinese herbs. 16th edition. SMC Publ. Inc., Taipei, pp451, 1989 (In Chinese).

25. Fasshauer M and Paschke R: Regulation of adipocytokines and insulin resistance. Diabetologia 46: 1594-1603, 2003.

26. Rosen ED and Spiegelman BM: Adipocytes as regulators of energy balance and glucose homeostasis. Nature 444: 847-853, 2006.

27. Shao D, Rangwala SM, Bailey ST, Krakow SL, Reginato MJ and Lazar MA: Interdomain communication regulating ligand binding by PPAR-gamma. Nature 396: 377-380, 1998.

28. Lefterova MI and Lazar MA: New developments in adipogenesis. Trends Endocrinol Metab 20: 107-114, 2009.

29. Havel PJ: Role of adipose tissue in body-weight regulation: Mechanisms regulating leptin production and energy balance. Proc Nutr Soc 59: 359-371, 2000.

30. Auwerx J and Staels B: Leptin. Lancet 351: 737-742, 1998.

31. Isaia GC, D'Amelio P, Di Bella S and Tamone C: Is leptin the link between fat and bone mass? J Endocrinol Invest 28 (Suppl 10): S61-S65, 2005.

32. Liu M and Liu F: Regulation of adiponectin multimerization, signaling and function. Best Pract Res Clin Endocrinol Metab 88: 25-31, 2014.

33. Axelsson J, Bergsten A, Qureshi AR, Heimbürger O, Bárány P, Lönnqvist F, Lindholm B, Nordfors L, Alvestrand A and Stenvinkel P: Elevated resistin levels in chronic kidney disease are associated with decreased glomerular filtration rate and inflammation, but not with insulin resistance. Kidney Int 69: 596-604, 2006

34. Lefterova MI, Zhang Y, Steger DJ, Schupp M, Schug J, Cristancho A, Feng D, Zhuo D, Stoeckert CJ Jr, Liu XS and Lazar MA: PPARgamma and C/EBP factors orchestrate adipocyte biology via adjacent binding on a genome-wide scale. Genes Dev 22: 2941-2952, 2008.

35. Tang QQ, Zhang JW and Daniel Lane M: Sequential gene promoter interactions of C/EBPbeta, C/EBPalpha, and PPARgamma during adipogenesis. Biochem Biophys Res Commun 319: 235-239, 2004

36. Rosen ED, Hsu CH, Wang X, Sakai S, Freeman MW, Gonzalez FJ and Spiegelman BM: C/EBPalpha, induces adipogenesis through PPARgamma: A unified pathway. Genes Dev 16: 22-26, 2002.

37. Schoonjans K, Staels B and Auwerx J: The peroxisome proliferator activated receptors (PPARS) and their effects on lipid metabolism and adipocyte differentiation. Biochim Biophys Acta 1302: 93-109, 1996.

38. Otto TC and Lane MD: Adipose development: From stem cell to adipocyte. Crit Rev Biochem Mol Biol 40: 229-242, 2005. 